fungal RNA induce protective immunity to Candida albicans in hematopoietic transplantation. J. Immunol. 168:2904-2913.

34. Asavaroengchai, W., Kotera, Y., and Mulé, J.J. 2002. Tumor lysate-pulsed dendritic cells can elicit an effective antitumor immune response during early lymphoid recovery. Proc. Natl. Acad. Sci. U. S. A. 99:931-936

35. Moyer, J.S., Maine, G., and Mulé, J.J. 2006. Early vaccination with tumor lysate-pulsed dendritic cells after allogeneic bone marrow transplantation has antitumor effects. Biol. Blood Marrow Transplant. 12:1010-1019.

36. Ge, Q., Hu, H., Eisen, H.N., and Chen, J. 2002. Different contributions of thymopoiesis and homeostasis-driven proliferation to the reconstitution of naive and memory T cell compartments. Proc. Natl. Acad. Sci. U. S. A. 99:2989-2994.

37. Mazo, I.B., et al. 2005. Bone marrow is a major reservoir and site of recruitment for central memory $\mathrm{CD}^{+} \mathrm{T}$ cells. Immunity. 22:259-270

38. Leen, A.M., et al. 2005. T-cell immunotherapy for adenoviral infections of stem-cell transplant recipients. Ann. N. Y. Acad. Sci. 1062:104-115.

39. Bollard, C.M., et al. 2006. In vivo expansion of LMP 1 - and 2 -specific T-cells in a patient who received donor-derived EBV-specific T-cells after allogeneic stem cell transplantation. Leuk. Lymphoma. 47:837-842.

40. Leen, A.M., et al. 2006. Monoculture-derived T lymphocytes specific for multiple viruses expand and produce clinically relevant effects in immunocom- promised individuals. Nat. Med. 12:1160-1166.

41. Riddell, S.R., Bleakley, M., Nishida, T., Berger, C. and Warren, E.H. 2006. Adoptive transfer of allogeneic antigen-specific T cells. Biol. Blood Marrow Transplant. 12:9-12.

42. Riddell, S.R., and Greenberg, P.D. 1997. T cell therapy of human CMV and EBV infection in immunocompromised hosts. Rev. Med. Virol. 7:181-192.

43. Kwak, L.W., Neelapu, S.S., and Bishop, M.R. 2004 Adoptive immunotherapy with antigen-specific $\mathrm{T}$ cells in myeloma: a model of tumor-specific donor lymphocyte infusion. Semin. Oncol. 31:37-46.

44. Neelapu, S.S., et al. 2005. Tumor antigen immunization of sibling stem cell transplant donors in multiple myeloma. Bone Marrow Transplant. 36:315-323

45. Rapoport, A.P., et al. 2005. Restoration of immunity in lymphopenic individuals with cancer by vaccination and adoptive T-cell transfer. Nat. Med. 11:1230-1237.

46. Teague, R.M., et al. 2006. Interleukin-15 rescues tolerant $\mathrm{CD}^{+} \mathrm{T}$ cells for use in adoptive immunotherapy of established tumors. Nat. Med. 12:335-341.

47. Tuma, R.A., Giannino, R., Guirnalda, P., Leinr, I., and Pamer, E.G. 2002. Rescue of CD8 T cellmediated antimicrobial immunity with a nonspecific inflammatory stimulus. J. Clin. Invest. 100:1493-1501.

48. Farrell, A. 2006. Defeating T-cell fatigue in HIV. Nat. Med. 12:1124-1125.

49. Overwijk, W.W., et al. 2003. Tumor regression and autoimmunity after reversal of a functionally toler- ant state of self-reactive CD8+ T cells. J. Exp. Med. 198:569-580.

50. Collin, M.P., et al. 2006. The fate of human Langerhans cells in hematopoietic stem cell transplantation. J. Exp. Med. 203:27-33.

51. Matte, C.C., et al. 2004. Donor APCs are required for maximal GVHD but not for GVL. Nat. Med. 10:987-992.

52. Reddy, P., et al. 2005. A crucial role for antigen-presenting cells and alloantigen expression in graft-versus-leukemia responses. Nat. Med. 11:1244-1249.

53. Feng, B., et al. 2000. Development of mouse dendritic cells from lineage-negative c-kit(low) pluripotent hemopoietic stem cells in vitro. Stem Cells. 18:53-60.

54. Ginhoux, F., et al. 2006. Langerhans cells arise from monocytes in vivo. Nat. Immunol. 7:265-273.

55. Merad, M., et al. 2004. Depletion of host Langerhans cells before transplantation of donor alloreactive $\mathrm{T}$ cells prevents skin graft-versus-host disease. Nat. Med. 10:510-517.

56. Zaft, T., Sapoznikov, A., Krauthgamer, R., Littman, D.R., and Jung, S. 2005. CD11c-high dendritic cell ablation impairs lymphopenia-driven proliferation of naive and memory $\mathrm{CD}^{+} \mathrm{T}$ cells. J. Immunol. 175:6428-6435.

57. Gattinoni, L., Powell, D.J., Rosenberg, S.A., and Restifo, N.P. 2006. Adoptive immunotherapy of cancer: building on success. Nat. Rev. Immunol. 6:383-393.

58. Morgan, R.A., et al. 2006. Cancer regression in patients after transfer of genetically engineered lymphocytes. Science. 314:126-129.

\title{
A link between protein translation and body weight
}

\section{Liangyou Rui}

\author{
Department of Molecular and Integrative Physiology, University of Michigan Medical School, Ann Arbor, Michigan, USA.
}

\begin{abstract}
Nutrient overload induces obesity, a primary risk factor for type 2 diabetes. Ribosomal biogenesis and protein synthesis, which are controlled by the mammalian target of rapamycin (mTOR), are primary energy-consuming processes in cells. mTOR phosphorylates and inactivates members of the eukaryotic translation initiation factor 4E-binding (eIF4E-binding) protein (4E-BP) family, which are translational repressors of $5^{\prime}$ cap-dependent protein synthesis. In this issue of the JCI, Le Bacquer et al. report that simultaneous deletion of both 4E-BP1 and 4E-BP2 in mice results in insulin resistance, decreased energy expenditure, and increased adipogenesis (see the related article beginning on page 387 ). These findings link protein synthesis, insulin sensitivity, and body weight.
\end{abstract}

Food (energy) shortage is a constant threat to the survival of a species. Individuals who can efficiently maintain their body weight via energy conservation have an increased

Nonstandard abbreviations used: DKO, double knockout; 4E-BP, eIF4E-binding protein; eIF, eukaryotic translation initiation factor; IRS-1, insulin receptor substrate 1 ; mTOR, mammalian target of rapamycin; mTORC, mTOR complex; PDK1, phosphoinositidedependent protein kinase 1; S6K, ribosomal protein S6 kinase; $5^{\prime}$ UTR, $5^{\prime}$ untranslated region.

Conflict of interest: The author has declared that no conflict of interest exists.

Citation for this article: J. Clin. Invest. 117:310-313 (2007). doi:10.1172/JCI31289. chance of survival and propagation during times when food supply is limited. Nutrients and hormones activate multiple evolutionarily conserved signaling pathways that govern the balance between energy intake and expenditure. Mammalian target of rapamycin (mTOR) is a well-conserved serine/threonine protein kinase that functions as an intracellular nutrient sensor to control protein synthesis, cell growth, and metabolism. In this issue of the JCI, Le Bacquer et al. demonstrate that the eukaryotic translation initiation factor 4E-binding (eIF4E-binding) protein (4E-BP) family of translational repressors, which are physiologic substrates of mTOR, play a key role in regulating body weight and glucose homeostasis in mice (1).

\section{mTOR regulation of energy and glucose metabolism}

mTOR is a member of the phosphoinositide kinase-related kinase family and is activated by nutrients (e.g., branched-chain amino acids) as well as by metabolic hormones, growth factors, and cytokines. mTOR binds to other regulatory components to form 2 distinct multiprotein complexes. The first complex, mTORC1, contains mTOR, regulator-associated protein of mTOR (Raptor), and $G$ protein $\beta$ subunit-like protein $(\mathrm{G} \beta \mathrm{L})$. The second complex, mTORC2, contains mTOR, rapamycin-insensitive companion of mTOR (Rictor), mammalian stress-activated protein kinase-interacting protein $1(\mathrm{mSin} 1)$, and $\mathrm{G} \beta \mathrm{L}$ (Figure 1). The adaptor proteins Raptor and Rictor determine the substrate specificity of mTORC1 and mTORC2, respectively. mTORC1 specifically phosphorylates ribosomal protein 


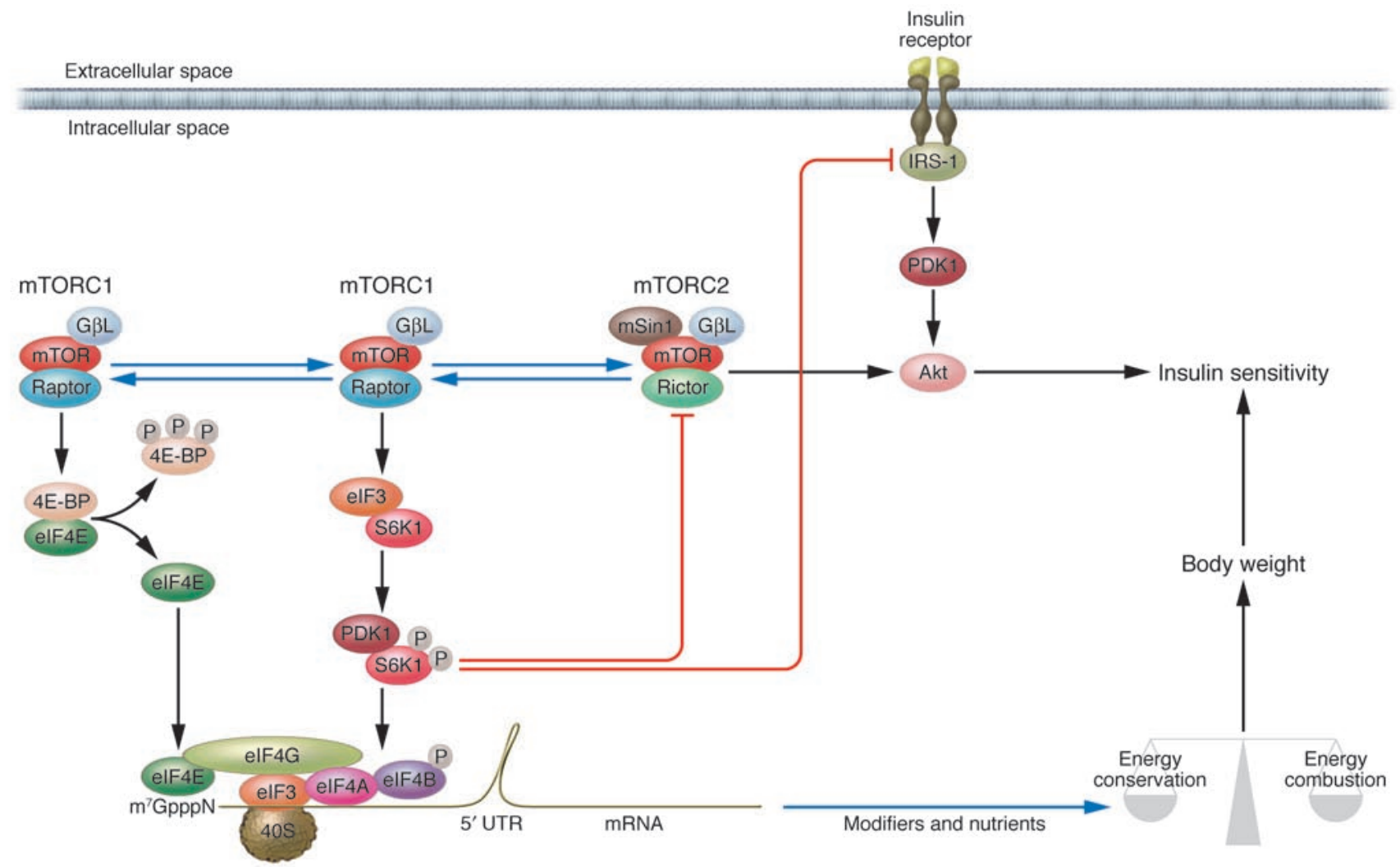

Figure 1

A model of the regulation of body weight and insulin sensitivity by mTOR, S6K1, and 4E-BP. Nutrients and hormones stimulate the association of $\mathrm{mTORC} 1$ with elF3. mTORC1 phosphorylates elF3-associated S6K1 at Thr389. Phosphorylated S6K1 is dissociated from elF3 and subsequently phosphorylated and activated by PDK1. S6K1 phosphorylates elF4B, increasing the ability of elF4B to enhance elF4A helicase activity. S6K1 phosphorylates IRS-1, inhibiting insulin signaling. S6K1 phosphorylates mTOR, which may decrease the abundance of mTORC2 and/or the ability of mTORC2 to phosphorylate Akt at Ser473, contributing to insulin resistance. A separate pool of mTORC1 may phosphorylate 4E-BP at multiple sites, resulting in dissociation of elF4E from 4E-BP. 4E-BP-free elF4E binds to elF4G that is associated with both elF4A and elF3. elF3 binds to the $40 \mathrm{~S}$ ribosomal subunit and recruits the $40 \mathrm{~S}$ subunit to the $5^{\prime}$ cap. elF4A, assisted by elF4B, unwinds the secondary structure in the mRNA $5^{\prime}$ UTR, facilitating the assembly of a translation initiation complex. The data reported in this issue by Le Bacquer et al. (1) suggest that reduced expression of 4E-BP increases the pool of mTORC1 that phosphorylates and activates S6K1. These data also raise the possibility that genetic modifiers and nutrients may modify the ability of 4E-BPs to repress the translation of key regulators of energy conservation and combustion programs, thereby regulating adiposity and body weight, which indirectly modulate insulin sensitivity. G $\beta \mathrm{L}, \mathrm{G}$ protein $\beta$ subunit-like protein; $\mathrm{m}^{7} \mathrm{GpppN}$, the $5^{\prime}$ cap structure; $\mathrm{mSin} 1$, mammalian stress-activated protein kinase interacting protein 1; Raptor, regulator-associated protein of mTOR; Rictor, rapamycin-insensitive companion of mTOR.

S6 kinase (S6K) and 4E-BP in a rapamycinsensitive manner. In contrast, mTORC2 phosphorylates Akt at Ser473, promoting Akt activation.

Thomas and colleagues have demonstrated that genetic deletion of S6K1, a physiologic substrate of mTORC1, results in profound metabolic defects in peripheral tissues (2-4). mTORC1 is required for adipocyte differentiation in vitro $(5,6)$. mTORC1 activity is significantly elevated in the livers, muscle, and adipose tissue of both genetically induced and diet-induced obese animals, suggesting the involvement of the peripheral mTORC1 pathway in the pathogenesis of obesity and obesity-associated metabolic disorders $(4,7)$. Cota, Seeley, and colleagues have demonstrated that the central mTORC1 pathway integrates various metabolic signals (both nutrients and hormones), thereby globally regulating energy homeostasis and body weight (8). They have shown that amino acids selectively activate the mTORC1 pathway in a subpopulation of hypothalamic neurons that control energy homeostasis, resulting in the inhibition of both energy intake and body weight gain (8). Conversely, inhibition of mTORC1 by rapamycin blocks amino acid- and leptininduced inhibition of energy intake and weight gain (8). Therefore, both the peripheral and the central mTORC1 pathways are required for maintaining normal energy and glucose metabolism.

\section{S6K1 regulation of energy and glucose metabolism}

mTORC1 phosphorylates S6K1 at Thr389, allowing the phosphoinositide-dependent protein kinase 1 (PDK1) to bind to and phosphorylate S6K1 at Thr229, thus fully activating S6K1 (9-11). S6K1 promotes protein synthesis and cell growth presumably by phosphorylating multiple substrates, including components of translation initiation and/or elongation machinery (e.g., ribosomal protein S6, eIF4B, and eukaryotic elongation factor 2 kinase). In animals, disruption of the $S 6 K 1$ gene reduces the size of pancreatic $\beta$ cells, resulting in insulin insufficiency and glucose intolerance (2). Moreover, S6K1 phosphorylates insu- 
lin receptor substrate 1 (IRS-1) at inhibitory serine/threonine residues, inhibiting insulin signaling (Figure 1). S6K1-deficient mice have enhanced insulin sensitivity (4). Interestingly, S6K1-deficient mice are protected from high-fat diet-induced obesity because of increased energy expenditure (4). Consistently, mitochondrial size and content increase in white adipocytes and skeletal muscle in S6K1-deficient mice. The expression of key molecules in fuel combustion, including uncoupling protein 1 (UCP1), UCP3, carnitine palmitoyltransferase 1 , and PPAR $\gamma$ coactivator $1 \alpha(\mathrm{PGC} 1 \alpha)$, is also increased in S6K1-deficient mice (4). Exactly how S6K1 regulates the program that controls energy combustion has remained unclear.

\section{E-BP-mediated regulation of energy and glucose metabolism}

The mammalian 4E-BP family has 3 members, 4E-BP1, 4E-BP2, and 4E-BP3, which repress protein translation by binding and inhibiting eIF4E (Figure 1). eIF4E directly binds to the mRNA 5' cap structure that is present in most eukaryotic mRNAs. eIF4E also binds to eIF4G, a scaffold protein that is associated with both eIF3 and eIF4A. eIF3 interacts with the $40 \mathrm{~S}$ ribosomal subunit, whereas eIF4A, an ATP-dependent mRNA helicase, unwinds the secondary structure in the mRNA $5^{\prime}$ untranslated region $\left(5^{\prime}\right.$ UTR) to facilitate the binding of the $40 \mathrm{~S}$ ribosomal subunit to the $5^{\prime}$ cap. Hypophosphorylated 4E-BPs binds with high affinity to eIF4E at sites overlapping with eIF4G, thereby inhibiting eIF4G binding and the assembly of a translation initiation complex at the mRNA 5' cap structure. mTORC1-induced hyperphosphorylation of 4E-BPs inhibits 4E-BP binding to eIF4E, which allows free eIF4E to bind to eIF4G and assemble a translation initiation complex at the mRNA $5^{\prime}$ cap, promoting $5^{\prime}$ capdependent protein synthesis (Figure 1).

To determine the physiologic functions of 4E-BP1 and 4E-BP2, Le Bacquer et al. (1) simultaneously deleted the genes coding for 4E-BP1 and 4E-BP2 in mice and examined the metabolic consequences. 4E-BP1 and 4E-BP2 double knockout (DKO) mice had a significant increase in both body weight and fat content. The obese phenotype was caused by reduced energy expenditure and reduced lipolysis. Not surprisingly, a highfat diet promoted insulin resistance to a greater degree in obese DKO mice than in WT mice. The metabolic phenotype is the opposite of that described for S6K1-defi- cient mice. Importantly, both embryonic fibroblasts and preadipocytes from DKO mice had an increased ability to differentiate into adipocytes in vitro; the expression of CCAAT/enchancer-binding protein $\delta$ (C/EBPS), C/EBP $\alpha$, and PPAR $\gamma$ - essential regulators for adipogenesis - was markedly increased in these cells in the DKO animals during differentiation. These observations suggest that 4E-BP1 and 4E-BP2 may inhibit adipocyte differentiation in a cell-autonomous fashion.

Interestingly, the authors demonstrated that S6K1 activation was significantly enhanced in multiple tissues in DKO mice (1). These findings suggest that not only do the parallel 4E-BP and S6K1 signaling pathways downstream of mTORC1 act coordinately in promoting translation, but that crosstalk exists between the 2 pathways, by which each pathway can modulate the activation of the other. Holz, Blenis, and colleagues have demonstrated that S6K1 binds to eIF3 under basal conditions (12). Insulin or amino acids promote mTORC1 binding to eIF3 and phosphorylation of S6K1 at Thr389 by eIF3-associated mTORC1 (Figure 1). This induces dissociation of S6K1 from eIF3, allowing PDK1 to bind to, phosphorylate, and activate S6K1 (12). Because eIF3 is associated with eIF4G, and eIF4G and 4E-BPs bind to eIF4E in a mutually exclusive manner, 4E-BPs may be unable to associate with eIF3 and be phosphorylated by eIF3-associated mTORC1. Therefore, 4E-BPs and S6K1 may be phosphorylated by 2 distinct pools of mTORC1, and the elimination of 4E-BPs may shift the balance of these 2 pools toward eIF3-associated mTORC1, resulting in increased activation of S6K1 in DKO mice (Figure 1). S6K1 induces insulin resistance, presumably by phosphorylation of IRS-1 at inhibitory serine/ threonine residues and degradation and transcriptional inhibition of IRS proteins in DKO mice. S6K1 also phosphorylates mTOR at multiple sites (13). This begs the question: Does S6K1-mediated phosphorylation modulate the ability of mTOR to assemble mTORC1 and mTORC2 and/or the ability of mTORC2 to phosphorylate and activate Akt?

Surprisingly, in Drosophila, the 4E-BP equivalent, d4E-BP, acts as a metabolic brake during stress by inhibiting the burning of energy (14). Consistent with this finding, Tsukiyama-Kohara, Sonenberg, and colleagues have previously examined 4E-BP1-deficient mice and reported that genetic deletion of 4E-BP1 protects against high-fat diet-induced obesity and insulin resistance because of increased energy expenditure (15). How can we reconcile these opposing metabolic phenotypes described in the current study and previous studies of 4E-BP1-deficient mice? Le Bacquer et al. (1) attribute the discrepancy to the differential expression of modifier genes, because the separate studies use 4E-BP1-deficient mice with 2 different genetic backgrounds (BALB/c 129SvJ1 in the earlier study versus $B A L B / c$ in the current study). Based on these assumptions, $4 \mathrm{E}$-BPs may repress the translation of key regulators that direct the programs of either energy conservation or energy combustion; a combination of genetic modifiers and environmental factors, especially nutrients, may determine the ability of 4E-BPs to repress energy conservation or combustion programs (Figure 1). The 5' cap structure and $5^{\prime}$ UTR of the mRNAs of these key regulators may control their translational efficiency in a $4 \mathrm{E}-\mathrm{BP}$ - and/or S6K1-dependent manner.

Because the activation of hypothalamic mTORC1 suppresses energy intake (8), it is surprising that deletion of neither S6K1 nor $4 \mathrm{E}-\mathrm{BP} 1$ or $4 \mathrm{E}-\mathrm{BP} 2$ alone is reported to alter food intake. Future studies may identify mTORC1 targets in the hypothalamus that crosstalk with other pathways (e.g., the JAK2/STAT3 and AMP kinase pathways) to mediate nutrient and/or hormonal regulation of energy homeostasis and body weight.

Address correspondence to: Liangyou Rui, Department of Molecular and Integrative Physiology, 7810 Med. Sci. II, 1301 Catherine Street, University of Michigan Medical School, Ann Arbor, Michigan 48109, USA. Phone: (734) 615-7544; Fax: (734) 647-9523; E-mail: ruily@umich.edu.

1. Le Bacquer, O., et al. 2007. Elevated sensitivity to diet-induced obesity and insulin resistance in mice lacking 4E-BP1 and 4E-BP2. J. Clin. Invest. 117:387-396. doi:10.1172/JCI29528.

2. Pende, M., et al. 2000. Hypoinsulinaemia, glucose intolerance and diminished beta-cell size in S6K1deficient mice. Nature. 408:994-997.

3. Shima, H., et al. 1998. Disruption of the p70(s6k)/ p85(s6k) gene reveals a small mouse phenotype and a new functional S6 kinase. $E M B O J$. 17:6649-6659.

4. Um, S.H., et al. 2004. Absence of S6K1 protects against age- and diet-induced obesity while enhancing insulin sensitivity. Nature. 431:200-205.

5. Kim, J.E., and Chen, J. 2004. Regulation of peroxisome proliferator-activated receptor-gamma activity by mammalian target of rapamycin and amino acids in adipogenesis. Diabetes. 53:2748-2756. 
6. Yeh, W.C., Bierer, B.E., and McKnight, S.L. 1995. Rapamycin inhibits clonal expansion and adipogenic differentiation of 3T3-L1 cells. Proc. Natl. Acad. Sci. U. S. A. 92:11086-11090.

7. Khamzina, L., Veilleux, A., Bergeron, S., and Marette, A. 2005. Increased activation of the mammalian target of rapamycin pathway in liver and skeletal muscle of obese rats: possible involvement in obesity-linked insulin resistance. Endocrinology. 146:1473-1481.

8. Cota, D., et al. 2006. Hypothalamic mTOR signaling regulates food intake. Science. 312:927-930.

9. Isotani, S., et al. 1999. Immunopurified mam- malian target of rapamycin phosphorylates and activates p70 S6 kinase alpha in vitro. J. Biol. Chem. 274:34493-34498.

10. Alessi, D.R., Kozlowski, M.T., Weng, Q.P., Morrice, N., and Avruch, J. 1998. 3-Phosphoinositide-dependent protein kinase 1 (PDK1) phosphorylates and activates the p70 S6 kinase in vivo and in vitro. Curr. Biol. 8:69-81.

11. Pullen, N., et al. 1998. Phosphorylation and activation of p70s6k by PDK1. Science. 279:707-710.

12. Holz, M.K., Ballif, B.A., Gygi, S.P., and Blenis, J. 2005. mTOR and S6K1 mediate assembly of the translation preinitiation complex through dynamic protein interchange and ordered phosphorylation events. Cell. 123:569-580.

13. Holz, M.K., and Blenis, J. 2005. Identification of S6 kinase 1 as a novel mammalian target of rapamycin (mTOR)-phosphorylating kinase. J. Biol. Chem. 280:26089-26093.

14. Teleman, A.A., Chen, Y.W., and Cohen, S.M. 2005 4E-BP functions as a metabolic brake used under stress conditions but not during normal growth. Genes Dev. 19:1844-1848.

15. Tsukiyama-Kohara, K., et al. 2001. Adipose tissue reduction in mice lacking the translational inhibitor 4E-BP1. Nat. Med. 7:1128-1132. 